\title{
Clinical Utility of MRI for Cholesteatoma Recurrence
}

\author{
Jonathan McJunkin • Richard Chole
}

Published online: 24 June 2014

(C) Springer Science + Business Media New York 2014

\begin{abstract}
Diffusion-weighted magnetic resonance imaging (DWI) has recently been proposed as an alternative to surgical exploration for suspected recurrent or residual cholesteatoma. A second-look surgery is often planned for extensive disease in ears where the posterior canal wall has been left intact. Alternatively, granulation tissue with chronic otorrhea in a patient with a history of cholesteatoma is highly suspicious for recurrence. Computerized tomography imaging is excellent for showing bony definition details of the temporal bone but cannot differentiate between soft tissue types. Cholesteatoma has unique signaling characteristics on DWI that can help distinguish between inflammatory tissue and residual or recurrent disease.
\end{abstract}

Keywords Cholesteatoma - Diffusion-weighted imaging · Recurrent cholesteatoma $\cdot$ Residual cholesteatoma $\cdot$ MRI

\section{Introduction}

Cholesteatoma is a benign lesion of the ear characterized by a capsule of squamous epithelium filled with keratinous debris. These lesions often demonstrate local bony erosion over time. The bony destruction is caused by osteoclast osteolysis induced by proinflammatory cytokines and

This article is part of the Topical Collection on Ear Surgery.

J. McJunkin $(\bowtie) \cdot$ R. Chole

Department of Otolaryngology, Washington University in St. Louis School of Medicine, 660 South Euclid Ave, St. Louis, MO 63110, USA

e-mail: mcjunkinj@ent.wustl.edu

R. Chole

e-mail: rchole@wustl.edu bacterial products [1]. Frequent presenting symptoms include chronic recurrent otorrhea and conductive hearing loss due to ossiclular erosion. Late and infrequent sequelae of cholesteatoma include vertigo and sensorineural hearing loss if the otic capsule is violated. Facial nerve palsy and meningitis can also occur [2].

Middle ear cholesteatomas can be classified as congenital or acquired. Congenital cholesteatomas develop from an embryonic remnant known as the epithelial rest. The lesion typically appears as a keratin pearl in the anterosuperior mesotympanum medial to an intact tympanic membrane in a patient without a history of recurrent ear infections or surgery. Congenital cholesteatomas are much less common than the acquired types.

There are two subtypes of acquired cholesteatomas: primary and secondary. Primary acquired cholesteatomas develop from tympanic membrane retraction; chronic eustachian tube dysfunction causes negative pressure in the middle ear space and retraction of the tympanic membrane. On otoscopic examination, a pars flacida retraction pocket filled with keratin debris and scutal erosion is typical (Fig. 1). Secondary acquired cholesteatomas develop after a perforation of the tympanic membrane.

\section{Management}

Cholesteatoma is typically managed by surgical removal. In patients unfit for surgery, the lesion can sometimes be evacuated assuming that the depths of the capsule can be reached. The primary goal of cholesteatoma surgery is the eradication of the disease and creating a healthy ear that will not be prone to recurrent infections. A secondary consideration is hearing restoration if there is conductive hearing loss due to disruption of the ossicles. Different 


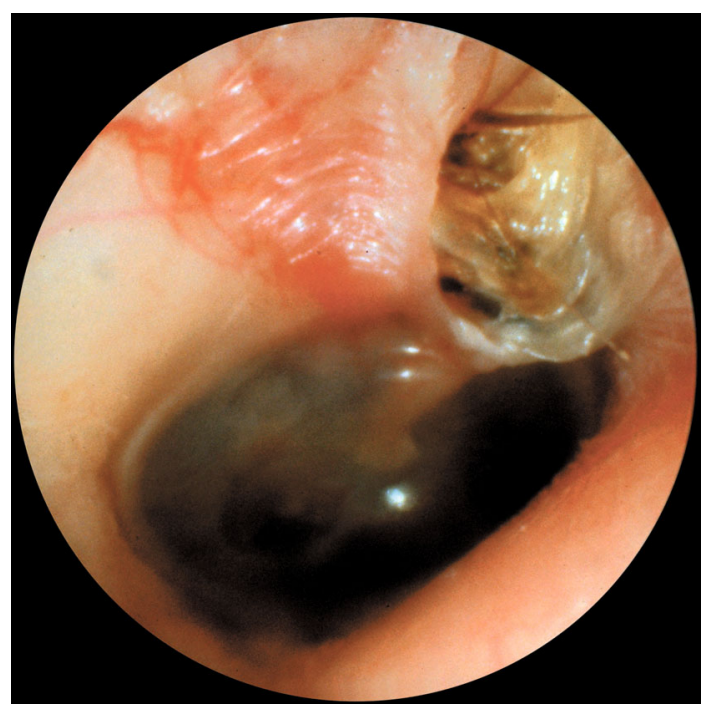

Fig. 1 Retraction pocket cholesteatoma of the pars flaccida portion of the tympanic membrane in a left ear. Note keratin debris filling the lesion

management strategies have been employed according to these principles.

One point of debate has been preservation of the posterior ear canal wall. Cholesteatomas that extend medial to the ossicular chain are more technically challenging to remove using a wall up approach. Some authors have advocated the use of endoscopes to inspect areas of the middle ear, which are difficult to visualize in a wall up approach such as the sinus tympani and the anterior epitympanum [3, 4]. Canal wall down surgery removes potential sites for cholesteatoma recurrence but takes longer to heal and can require more frequent cleaning.

Some authors also advocate the use of a planned "second look" after intact canal wall surgery. For extensive disease or inflammation, a staged operation (usually at least 6 months) after the initial cholesteatoma operation is used to check for residual cholesteatoma and reconstruct the ossicular chain if indicated. Recurrent disease is typically identified with binocular microscopy in the clinic. However, some patients will have recurrent infectious symptoms that can be hard to attribute to recurrent cholesteatoma or granulation tissue.

Recently, diffusion-weighted imaging MRI (DWI) has been proposed as an alternative to second-look surgery or situations of suspected cholesteatoma recurrence.

\section{Imaging Characteristics of Cholesteatoma}

High-resolution computed tomography (HRCT) is very useful in the imaging of cholesteatoma [5•]. HRCT accurately delineates bony erosion caused by cholesteatoma and helps evaluate the anatomy and status of the ossicles, scutum, labyrinth, tegmen, and other surrounding structures. These scans can be obtained quickly without intravenous contrast and little patient discomfort. HRCT is routinely used in the initial workup of patients with newly diagnosed cholesteatoma. These keratin-filled lesions appear as a soft tissue density on HRCT. However, HRCT cannot differentiate well between cholesteatoma, fluid, or granulation tissue. CT has poor sensitivity and specificity for determining the presence of residual or recurrent cholesteatoma [6].

MRI is more useful for evaluating soft tissue details. Cholesteatoma tissue appears dark on T1-weighted images, bright on T2-weighted images, and does not enhance with gadolinium. To further distinguish keratin cysts from other tissue types, delayed postgadolinium MRI(DPI) has been utilized as other tissues will enhance with contrast over time [7]. Images are obtained 30-45 min after contrast administration. Ayache [6] reported $90 \%$ sensitivity and $100 \%$ specificity when using DPI for detection of residual cholesteatoma after canal wall up surgery. Lesions smaller than $3 \mathrm{~mm}$ were not detected on MRI. Disadvantages of DPI include the time and expense associated with the gadolinium use. The time factor becomes a particular concern in the pediatric population where MRI scans are frequently done under general sedation to minimize movement.

\section{Use of Diffusion-Weighted MRI for Cholesteatoma Management}

DWI is based on detecting molecular diffusion, random movement of water particles, in different tissue states. Water molecules with restricted movement are associated with higher signal intensity. Tumor and ischemic tissue have restricted molecular diffusion and high signal intensity on DWI. Epidermoids also have high signal intensity on DWI that is postulated to be due to either diffusion restriction or T2 shine-through effect [8]. The keratin within the lesion causes diffusion restriction. Other tissues suspicious for cholesteatoma on HRCT such as brain, fibrosis, edema, and granulation tissue have low signal intensity on DWI. Therefore, DWI signal intensity can be used to help distinguish between cholesteatoma and other tissue types (Table 1).

There are two types of DWI: echo-planar imaging (EPI) and "non-EPI." EPI was described first and is done with a single-shot spin echo pulse sequence. Multiple studies have described using EPI imaging to detect cholesteatoma [714]. Vercruysse [13] studied EPI in 55 patients with presumed primary cholesteatoma and 45 patients undergoing second-look surgery. For the primary group, EPI had $81 \%$ 
Table 1 MRI characteristics of cholesteatoma versus granulation tissue

\begin{tabular}{lll}
\hline Sequence & Cholesteatoma & $\begin{array}{l}\text { Granulation } \\
\text { tissue }\end{array}$ \\
\hline $\mathrm{T} 1$ & Intermediate signal & $\begin{array}{l}\text { Intermediate } \\
\text { signal } \\
\text { Tntermediate }\end{array}$ \\
$\begin{array}{l}\text { Post gadolinium } \\
\begin{array}{l}\text { Delayed post } \\
\text { gadolinium } \\
\text { Diffusion weighted }\end{array}\end{array}$ & $\begin{array}{l}\text { No enhancement } \\
\text { No enhancement }\end{array}$ & Enhancement \\
& Enhancement \\
& diffusion) & Dark \\
\hline
\end{tabular}

sensitivity and $100 \%$ specificity. The false negative cases were found to be retraction pocket lesions with little or no keratin accumulation. In the second-look surgery group, EPI correctly identified residual disease in only one of seven patients ( $12 \%$ sensitivity). The residual lesions were all less than $4 \mathrm{~mm}$ with the exception of the lesion identified on EPI, which was $6 \mathrm{~mm}$. Jindal [11] demonstrated $83 \%$ sensitivity and $82 \%$ specificity for EPI for the diagnosis of residual cholesteatoma. The disadvantage of the EPI modality is the presence of artifacts caused by tissue interfaces that can be misinterpreted as recurrent disease [15]. There are multiple tissue interfaces in the middle ear and mastoid that make these artifacts problematic.

The non-EPI DWI modality utilizes either multishot turbo-spin sequences [Periodically Rotated Overlapping Parallel Lines with Enhanced Reconstruction (PROPELLER); BLADE (Siemens Systems, Germany)] or single-shot turbo-spin sequences[(HASTE: Half Fourier Acquisition
Single-Shot Turbo-Spin Echo, (Siemens Systems, Germany)] [5]. The non-EPI modalities reduce interface artifacts and have better image quality due to improved spatial resolution [11]. Various studies have examined the utility of this imaging modality for cholesteatoma [16-21, 22•, 23•, 24-26]. In one systematic review, Jindal [22•] found nonEPI to be superior to EPI with sensitivity and specificity of 91.4 and $95.8 \%$ compared to 70.6 and $87.3 \%$. Li [23•] et al. performed a meta-analysis of non-EPI and found $94 \%$ sensitivity and specificity for non-EPI to identify cholesteatoma. Surgery was used as the gold standard to confirm the presence of cholesteatoma. Ten studies and 342 patients were analysed in the meta-analysis. False negative cases were typically cholesteatomas $3 \mathrm{~mm}$ or smaller. False positive results were due to cholesterol granuloma, susceptibility artifacts, abscess, or bone powder.

\section{Case Presentation 1}

A 42-year-old female with a history of left cholesteatoma and multiple operations presented to clinic with pain, swelling, and edema over her left mastoid tip for several days. Her hearing was not affected, and she was otherwise neurologically intact. On examination, she had a wellhealed mastoid cavity and intact tympanic membrane. The middle ear space was clear. The abscess was incised and drained; the fluid culture was positive for methicillinresistant Staphylococcus aureus. After her infection had resolved, HRCT imaging was obtained which showed a soft tissue density in her mastoid tip (Fig. 2). A DWI was ordered to investigate the nature of the soft tissue given the
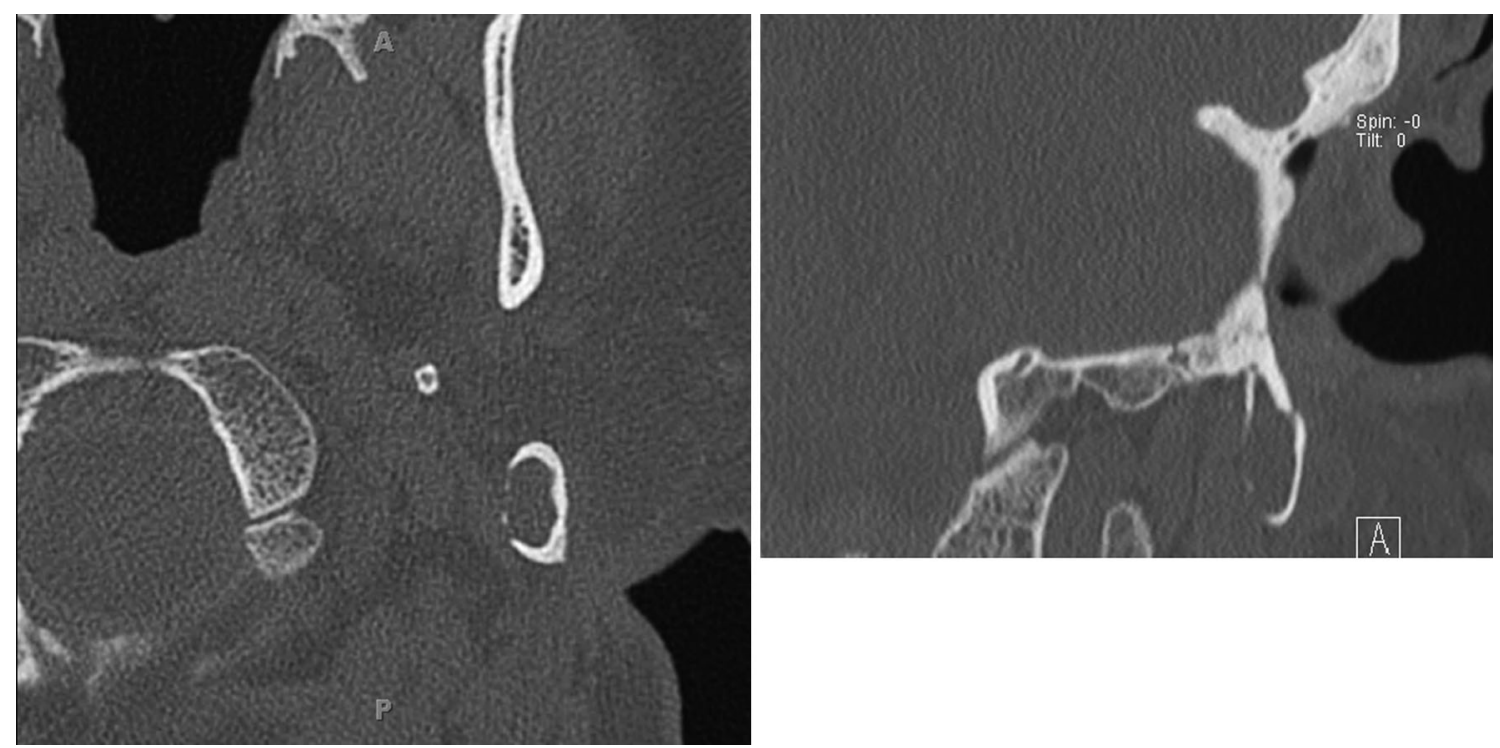

Fig. 2 HRCT coronal and axial views of a soft tissue lesion present in the left mastoid tip. CT cannot distinguish between cholesteatoma and inflammatory tissue 

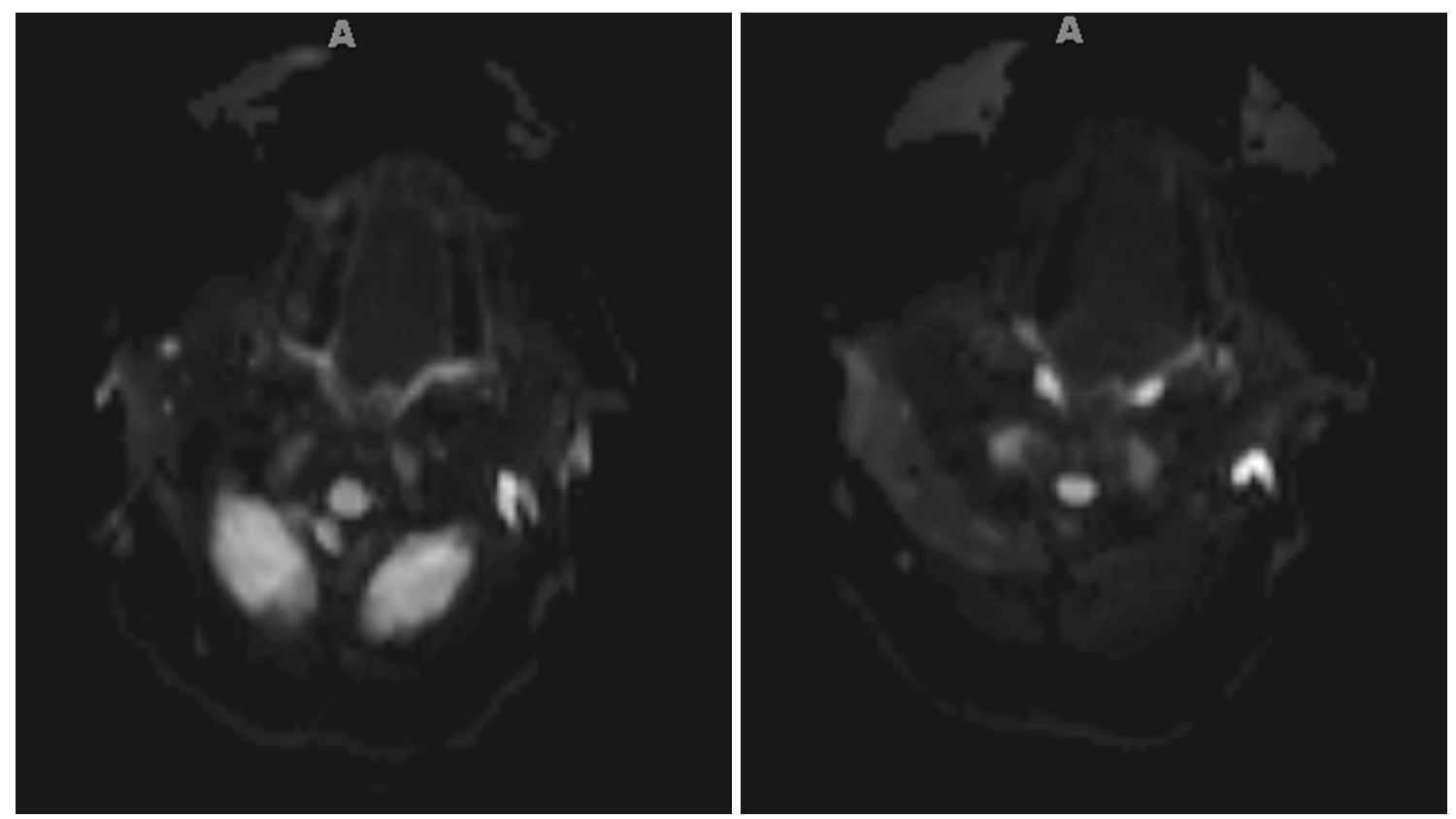

Fig. 3 Diffusion-weighted images (EPI) showing high destruction refusion over the last mastoid tip consistent with keratin debris representing a cholesteatoma

innocuous appearance of her mastoid cavity (Fig. 3). The $1 \mathrm{~cm}$ area of soft tissue density in the left mastoid tip demonstrated diffusion restriction consistent with recurrent cholesteatoma. She was taken to the operating room for revision mastoidectomy. She was found to have recurrent cholesteatoma confined to the mastoid tip that did not communicate with her cavity. The mastoid tip and cholesteatoma were removed without disturbing the middle ear. She healed well and has had no evidence of recurrence to date.

\section{Case Presentation 2}

A 43-year-old male with history of canal wall up left tympanomastoidectomy for attic cholesteatoma 5 years prior presented to clinic with recurrent otorrhea and muffled hearing. The tympanic membrane had been reconstructed with cartilage at the initial operation. On exam, there was granulation tissue with mucopurulent drainage over the posterosuperior drum. The granulation tissue cleared after cleaning and antibiotic drops for 1 week (Fig. 4). However, the patient returned 2 months later with recurrent symptoms. A CT scan demonstrated opacification of the middle ear space and mastoid. Diffusion-weighted imaging demonstrated a non-enhancing lesion in the epitympanum with diffusion restriction consistent with cholesteatoma (Fig. 5). The patient underwent a canal wall down tympanomastoidectomy and cholesteatoma was

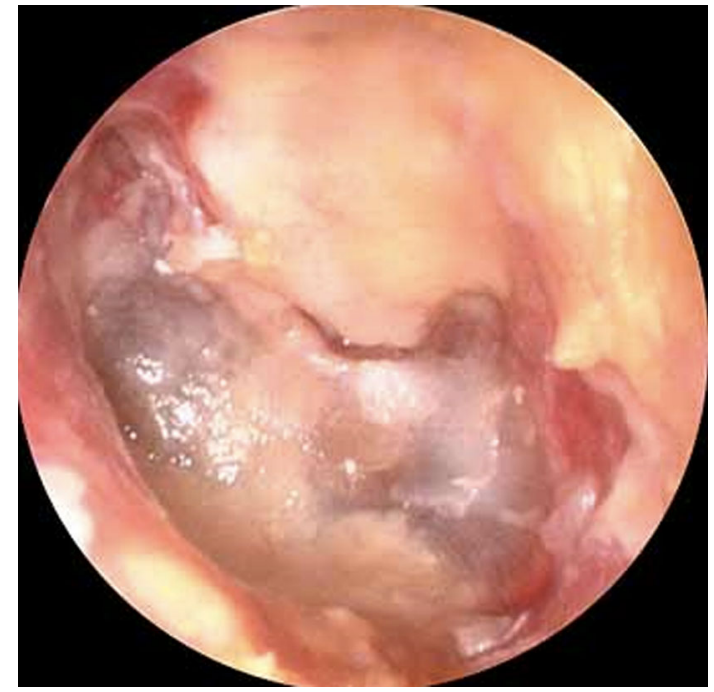

Fig. 4 Case 2 otoscopic exam demonstrating cartilage reconstruction of the posterosuperior tympanic membrane and resolving granulation tissue

removed from the antrum and epitympanum corresponding well with the DWI findings.

\section{Discussion}

At the author's institution, cholesteatoma is initially diagnosed based on clinical history and physical exam findings. HRCT imaging is frequently obtained to demonstrate the 

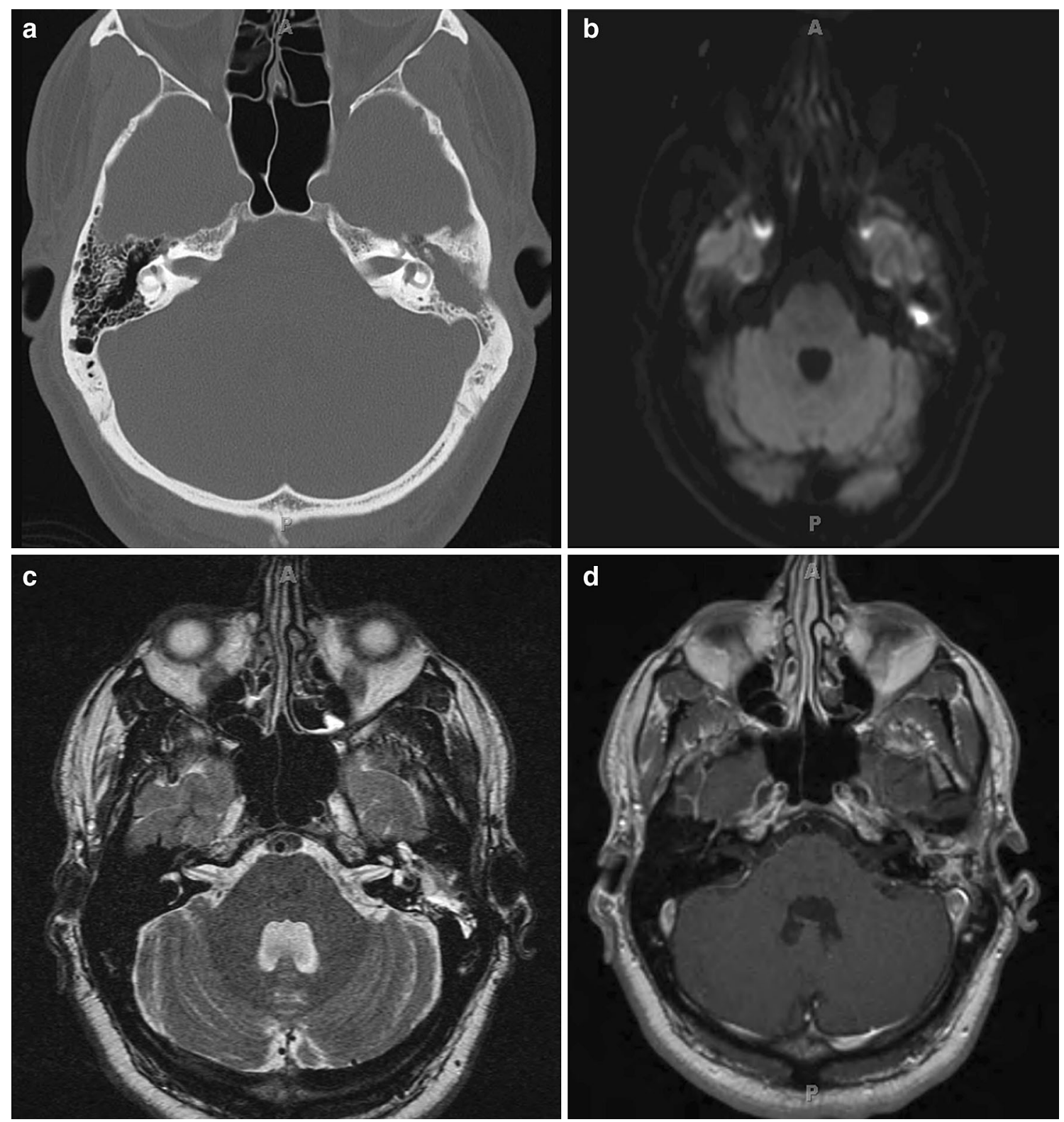

Fig. 5 (a) Axial CT: opacification of the middle ear and antrum. (b) DW MRI: area of restricted diffusion overlying the antrum consistent with keratin debris within a cholesteatoma. (c) T2 MRI:

extent of the lesion and relevant temporal bone anatomy. Conventional MRI is typically reserved for cases of suspected intracranial involvement. Surgical management is dictated by each case, and both canal wall up and canal wall down techniques are used. Second-look surgery is not routinely utilized except in cases of suspected residual disease or for reconstruction of the ossicular chain. Routine follow-up is mandatory for clinical surveillance of cholesteatoma recurrence. DWI is typically reserved for difficult cases in symptomatic patients that have suspected recurrent cholesteatoma that is not readily apparent on exam. soft tissue density noted lateral to the lateral semicircular canal. (d) T1 with Gad MRI: soft tissue density does not enhance

It is important to note that the current literature on DWI of cholesteatoma is not uniform in the imaging modalities applied. Jindal [22•] astutely noted several confounding variables when assessing the DWI literature. Different centers use different imaging parameters as there are no uniform settings when interpreting diffusion images. Additionally, Jindal notes in his review: "the incidence of recurrent or residual disease varies widely from 7 to $78 \%$ " [22-]. The incidence of a disease will strongly influence the statistical strength of any screening test. While non-EPI has been shown to be superior to EPI due to less artifact and 
better resolution, not all centers currently utilize the nonEPI modality.

The two-case presentations above demonstrate ideal patients for diffusion-weighted imaging. The first patient had a benign ear exam but recurrent skin infections over the mastoid tip region. CT imaging demonstrated a nonspecific soft tissue lesion, while the DWI confirmed the suspicion of cholesteatoma. The second patient had recurrent otorrhea but identifying the cholesteatoma was difficult given the cartilage reconstruction obscuring the attic. Cartilage is ideal for reconstruction of the tympanic membrane and scutum in patients with cholesteatoma as it provides rigidity to prevent future retraction.

Diffusion-weighted imaging is most useful in cases of uncertain cholesteatoma recurrence. Specifically, patients with previous wall up operations and resulting obscured anatomy or recurrent ear infections without keratin evident on binocular microscopy. Given this patient, population typically has underlying Eustachian tube dysfunction, granulation, and drainage can occur without cholesteatoma. DWI can be used in cases where a second-look operation would typically be performed given its high sensitivity for detecting recurrence. Future research to demonstrate the efficacy of this imaging modality should include long-term follow-up.

\section{Conclusions}

DWI is a useful adjunctive diagnostic imaging modality for the identification of residual or recurrent cholesteatoma. The non-EPI modality is superior to EPI and delayed postgadolinium techniques. Limitations include lesions less than $3 \mathrm{~mm}$ in diameter. DWI could potentially preclude unnecessary second-look surgery for cholesteatoma.

\section{Compliance with Ethics Guidelines}

Conflict of Interest Jonathan McJunkin and Richard Chole declare that they have no conflicts of interest.

Human and Animal Rights and Informed Consent This article does not contain any studies with human or animal subjects performed by the authors.

\section{References}

Papers of particular interest, published recently (within 3 years), have been highlighted as:

- Of importance

1. Jung JY, Chole RA. Bone resorption in chronic otitis media: the role of the osteoclast. ORL J Otorhinolaryngol Relat Spec. 2002;64(2):95-107.
2. Merchant SNJ, Schucknecht's Pathology of the Ear. In: Merchant S, editor. 3rd ed. People's Medical Publishing House: USA; 2010.

3. Ayache S, Tramier B, Strunski V. Otoendoscopy in cholesteatoma surgery of the middle ear: what benefits can be expected? Otol Neurotol. 2008;29(8):1085-90.

4. Tarabichi M, et al. Transcanal endoscopic management of cholesteatoma. Otolaryngol Clin North Am. 2013;46(2):107-30.

5. - Corrales, CE, NH Blevins. Imaging for evaluation of cholesteatoma: current concepts and future directions. Curr Opin Otolaryngol Head Neck Surg. 2013;21(5): 461-7. Comprehensive review and comparison of $C T$ and MRI for cholesteatoma management.

6. Ayache D, et al. Imaging of non-operated cholesteatoma: clinical practice guidelines. Eur Ann Otorhinolaryngol Head Neck Dis. 2012;129(3):148-52.

7. Venail F, et al. Comparison of echo-planar diffusion-weighted imaging and delayed postcontrast T1-weighted MR imaging for the detection of residual cholesteatoma. AJNR Am J Neuroradiol. 2008;29(7):1363-8.

8. Aikele P, et al. Diffusion-weighted MR imaging of cholesteatoma in pediatric and adult patients who have undergone middle ear surgery. AJR Am J Roentgenol. 2003;181(1):261-5.

9. Cimsit NC, et al. Diffusion-weighted MR imaging in postoperative follow-up: reliability for detection of recurrent cholesteatoma. Eur J Radiol. 2010;74(1):121-3.

10. Jeunen $\mathrm{G}$, et al. The value of magnetic resonance imaging in the diagnosis of residual or recurrent acquired cholesteatoma after canal wall-up tympanoplasty. Otol Neurotol. 2008;29(1):16-8.

11. Jindal $\mathrm{M}$, et al. Diffusion-weighted magnetic resonance imaging in the management of cholesteatoma. Eur Arch Otorhinolaryngol. 2010;267(2):181-5.

12. Stasolla A, et al. Value of echo-planar diffusion-weighted MRI in the detection of secondary and postoperative relapsing/residual cholesteatoma. Radiol Med. 2004;107(5-6):556-68.

13. Vercruysse JP, et al. The value of diffusion-weighted MR imaging in the diagnosis of primary acquired and residual cholesteatoma: a surgical verified study of 100 patients. Eur Radiol. 2006;16(7):1461-7.

14. Lehmann $P$, et al. $3 T$ MR imaging of postoperative recurrent middle ear cholesteatomas: value of periodically rotated overlapping parallel line with enhanced reconstruction diffusionweighted MR imaging. AJNR Am J Neuroradiol. 2009;30(2): 423-7.

15. Patel, Klufas R, Alberico RA, Edelman RR. Half-Fourier acquisition single-shot turbo spine-echo (HASTE) MR: comparison with fast spin-echo MR in diseases of the brain. AJNR Am J Neuroradiol. 1997;18(9):1635-40.

16. De Foer B, et al. Middle ear cholesteatoma: non-echo-planar diffusion-weighted MR imaging versus delayed gadoliniumenhanced T1-weighted MR imaging-value in detection. Radiology. 2010;255(3):866-72.

17. Dhepnorrarat RC, Wood B, Rajan GP. Postoperative non-echoplanar diffusion-weighted magnetic resonance imaging changes after cholesteatoma surgery: implications for cholesteatoma screening. Otol Neurotol. 2009;30(1):54-8.

18. Dubrulle F, et al. Diffusion-weighted MR imaging sequence in the detection of postoperative recurrent cholesteatoma. Radiology. 2006;238(2):604-10.

19. Edfeldt L, et al. Non-echo planar diffusion-weighted MRI increases follow-up accuracy after one-step step canal wall-down obliteration surgery for cholesteatoma. Acta Otolaryngol. 2013;133(6):574-83.

20. Geoffray A, et al. MRI for the diagnosis of recurrent middle ear cholesteatoma in children-can we optimize the technique? Preliminary study. Pediatr Radiol. 2013;43(4):464-73. 
21. Huins CT, et al. Detecting cholesteatoma with non-echo planar (HASTE) diffusion-weighted magnetic resonance imaging. Otolaryngol Head Neck Surg. 2010;143(1):141-6.

22. - Jindal M., et al. A systematic review of diffusion-weighted magnetic resonance imaging in the assessment of postoperative cholesteatoma. Otol Neurotol. 2011. 32(8): 1243-9. A well written review article that summarizes and explains DWI and its limitations.

23. - Li, P.M., et al. Evaluating the utility of non-echo-planar diffusion-weighted imaging in the preoperative evaluation of cholesteatoma: a meta-analysis. Laryngoscope. 2013. 123(5): 1247-50. Meta-analysis assessing the utility of preoperative DWI for detection of cholesteatoma.
24. Pizzini FB, et al. HASTE diffusion-weighted 3-Tesla magnetic resonance imaging in the diagnosis of primary and relapsing cholesteatoma. Otol Neurotol. 2010;31(4):596-602.

25. Plouin-Gaudon I, et al. Fusion of MRIs and CT scans for surgical treatment of cholesteatoma of the middle ear in children. Arch Otolaryngol Head Neck Surg. 2010;136(9):878-83.

26. Rajan GP, et al. Preliminary outcomes of cholesteatoma screening in children using non-echo-planar diffusion-weighted magnetic resonance imaging. Int $\mathrm{J}$ Pediatr Otorhinolaryngol. 2010;74(3):297-301. 